\title{
Responsiveness of platelets during storage studied with flow cytometry - formation of platelet subpopulations and LAMP-1 as new markers for the platelet storage lesion
}

\author{
Anna Södergren, Nahreen Tynngård, Gösta Berlin and Sofia Ramström
}

\section{Linköping University Post Print}

\section{Tweet}

N.B.: When citing this work, cite the original article.

Original Publication:

Anna Södergren, Nahreen Tynngård, Gösta Berlin and Sofia Ramström, Responsiveness of platelets during storage studied with flow cytometry - formation of platelet subpopulations and LAMP-1 as new markers for the platelet storage lesion, 2016, Vox Sanguinis, (110), 2, 116125.

http://dx.doi.org/10.1111/vox.12324

Copyright: Wiley: 12 months

http://eu.wiley.com/WileyCDA/

Postprint available at: Linköping University Electronic Press

http://urn.kb.se/resolve?urn=urn:nbn:se:liu:diva-126141 


\title{
Responsiveness of platelets during storage studied with flow cytometry - formation of platelet subpopulations and LAMP-1 as new markers for the platelet storage lesion
}

\author{
Anna L Södergren ${ }^{1}$, Nahreen Tynngård ${ }^{2,3}$, Gösta Berlin $^{2}, \&$ Sofia Ramström ${ }^{1}$ \\ ${ }^{1}$ Department of Clinical and Experimental Medicine, Linköping University, Linköping, \\ Sweden \\ ${ }^{2}$ Department of Clinical Immunology and Transfusion Medicine, and Department of Clinical \\ and Experimental Medicine, Linköping University, Linköping, Sweden \\ ${ }^{3}$ Department of Clinical Chemistry and Department of Clinical and Experimental Medicine, \\ Linköping University, Linköping, Sweden
}

Correspondence to: Sofia Ramström

Department of Clinical and Experimental Medicine,

Clinical Chemistry, Faculty of Health Sciences

Linköping University

SE-58185 Linköping, Sweden

Telephone:+46 101038600 Fax:+46 1010333240

e-mail:sofia.ramstrom@liu.se

Short title: Platelet subpopulations and LAMP-1 in stored platelets

Key words: Apheresis, hemostasis, Platelet concentrates, Platelet function, Platelet

transfusion 


\begin{abstract}
Background and Objectives: Storage lesions may prevent transfused platelets to respond to agonists and arrest bleeding. The aim of this study was to evaluate and quantify the capacity of platelet activation during storage using flow cytometry and new markers of platelet activation.
\end{abstract}

Materials and Methods: Activation responses of platelets prepared by apheresis were measured on days 1, 5, 7 and 12. In addition comparisons were made for platelet concentrates stored until swirling was affected. Lysosome associated membrane protein-1 (LAMP-1), Pselectin and phosphatidylserine (PS) exposure was assessed by flow cytometry on platelets in different subpopulations in resting state or following stimulation with platelet agonists (crosslinked collagen-related peptide (CRP-XL), PAR1- and PAR4-activating peptides).

Results: The ability to form subpopulations upon activation was significantly decreased already at day 5 for some agonist combinations. The agonist-induced exposure of PS and LAMP-1 also gradually decreased with time. Spontaneous exposure of P-selectin and PS increased with time, while spontaneous LAMP-1 exposure was unchanged. In addition, agonist-induced LAMP-1 expression clearly discriminated platelet concentrates with reduced swirling from those with retained swirling. This suggests that LAMP-1 could be a good marker to capture changes in activation capacity in stored platelets.

Conclusion: The platelet activation potential seen as LAMP-1 exposure and fragmentation into platelet subpopulations are potential sensitive markers for the platelet storage lesion. 


\section{Introduction}

To seal damaged blood vessels and prevent bleeding, platelets will adhere to proteins exposed in the subendothelium. This results in platelet activation, shape change, secretion from granules (alpha, dense and lysosomes) and exposure of phosphatidylserine (PS) to create a "procoagulant" surface that allows binding of coagulation factors. This procedure also involves membrane "blebbing" and formation of "microparticles" [1]. It is increasingly recognised that only a fraction of strongly activated platelets will expose PS, suggesting that platelets may take on different roles in haemostasis, but the underlying mechanisms are still largely unexplored (reviewed in [2, 3]).

Storage of platelet concentrates (PCs) results in the platelet storage lesion including changes in metabolism, surface receptors and response towards activation, as reviewed by Cauwenberghs et al. and Ohto et al. [4, 5]. Quality testing of PCs often involves analysis by several in vitro methods as surrogate markers for in vivo platelet viability [6,7] including analysis of blood gases, metabolic variables and aggregation using light transmission $[7,8]$ or impedance aggregometry (i.e. Multiplate) $[9,10]$. Spontaneous expression of activation markers, including P-selectin released from alpha-granules and the apoptosis marker PS, are commonly assessed using flow cytometry. Microparticle formation has also been investigated [11]

Lysosomal proteins such as lysosome associated membrane protein-1 (LAMP-1) become exposed on the platelet surface upon platelet activation, indicating that lysosomes are secreted under certain conditions [12]. Surface expression of LAMP-1, LAMP-2 and LIMP (LAMP3/CD63) was also reported in platelets in blood obtained from a bleeding wound, indicating that lysosomal exocytosis occur "in vivo" [13]. The role of lysosomal exocytosis in platelets is 
still unclear but might be involved in the clearance of thrombi, vessel wall remodelling and modulation of platelet membrane proteins $[13,14]$. To the best of our knowledge, no previous studies have investigated the release of markers from lysosomes during storage of PCs or the agonist-induced formation of platelet subpopulations. The aim of this study was to quantify how platelet storage affects the formation of platelet subpopulations and the expression of LAMP-1, PS and P-selectin using flow cytometry. 


\section{Methods}

\section{Platelet collection, preparation, storage and sampling}

Platelets were collected by standard apheresis procedures from 6 healthy donors (COBE Spectra ${ }^{\circledR}, \mathrm{n}=4$ and Trima Accel ${ }^{\circledR}, \mathrm{n}=2$, Terumo BCT, Lakewood, CO, USA). Acid citrate dextrose was used as anticoagulant [15]. The PC units contained $288 \pm 28 \times 10^{9}$ platelets on the day of donation (day 0). All PCs were irradiated with 25Gy on day 0 (Gammacell 3000 Elan, MDS Nordion, Ottawa, Canada). The PCs were stored in bags integrated to each collection kit on an agitator (Model LPR-3, Melco Engineering, Glendale, CA, USA) in an incubator (Helmer PC2200, Helmer, Noblesville, IN, USA) with a stable temperature of $22 \pm 2{ }^{\circ} \mathrm{C}$. Samples were taken aseptically with a sampling bag (MacoPharma, Tourcoing, France) from each PC on days 1, 5, 7 and 12 (one PC on day 13 for flow cytometry measurements). One PC (prepared by COBE Spectra) had reduced swirling on day 12, thus as a comparison we performed flow cytometry measurements on samples from 5 additional PCs with decreased swirling (on day 15-22, collected by apheresis Trima Accel ${ }^{\circledR}, \mathrm{n}=3$ and Spectra Optia ${ }^{\circledR}, \mathrm{n}=2$, Terumo BCT). The eBDS system was used to check for bacterial growth (Haemonetics Corporation, Braintree, MA, USA).

\section{Examination of basal platelet concentrate characteristics}

Swirling was examined visually [16] and graded as $0,+$, or $+++(0=$ no swirling, +++ =maximum swirling). Platelet concentration and mean platelet volume (MPV) were measured on a CELL-DYN-Sapphire haematology analyser (Abbott Diagnostics Division, Abbot Park, IL, USA). $\mathrm{pH}$ was measured at $37^{\circ} \mathrm{C}$ with a blood gas analyser (Cobas b221, Roche, Diagnostics GmbH, Mannheim, Germany). Glucose, lactate and lactate dehydrogenase (LDH) were measured with a chemistry analyser (Advia 1800, Siemens AG, Erlangen, Germany). 


\section{Flow cytometry}

Expression of the surface receptor GPIb and activation markers P-selectin, LAMP-1 and PS was analysed with a Gallios ${ }^{\mathrm{TM}}$ flow cytometer (Beckman Coulter Inc., Fullerton, CA, USA). Fluorescent control beads (Flow-Check and Flow-Set, Beckman Coulter Inc.) were used to verify that the instrument performance was stable over time.

Antibodies used were anti-GPIIb-PE (CD41a, clone: HIP8, final concentration $0.03 \mu \mathrm{g} / \mathrm{mL}$ ) and anti-P-selectin-PE-Cy5 (CD62P, clone: AK-4, 1 $\mu \mathrm{g} / \mathrm{ml})$ or anti-GPIIb-PE, anti-LAMP-1PE-Cy5 (CD107a, clone: H4A3, 0.5 $\mu \mathrm{g} / \mathrm{ml}$ ) and annexin V-FITC (binds to PS, $0.1 \mu \mathrm{g} / \mathrm{ml}$ ). As negative control for P-selectin and LAMP-1, a PE-Cy5-conjugated $\mathrm{IgG}_{1}$ isotype control antibody with matching concentration was used. As negative control for Annexin V HEPES buffer without added calcium was used in all steps throughout the procedure. Antibodies listed above were from BD Biosciences, San Jose, CA, USA. GPIb expression was analysed using anti-GPIb $\alpha$-FITC (CD42b, clone: AN51, $3.8 \mu \mathrm{g} / \mathrm{ml})$ from Dako, Glostrup, Denmark

Agonists used to stimulate platelets were cross-linked collagen-related peptide (CRP-XL [17], final concentration $0.9 \mu \mathrm{g} / \mathrm{ml}$, purchased from Richard Farndale, University of Cambridge, Cambridge, UK), protease activated receptor 1-activating peptide (PAR1-AP, amino acid sequence SFLLRN, 30 $\mu \mathrm{M}$ ), and PAR4-AP (amino acid sequence AYPGKF, 150 $\mu \mathrm{M}$, both from JPT Peptide Technologies GmbH, Berlin, Germany). Agonists were used alone or in combination (i.e. CRP-XL, PAR1-AP, PAR4-AP, CRP-XL+PAR1-AP, CRP-XL+PAR4-AP, PAR1-AP+PAR4-AP, CRP-XL+PAR1-AP+PAR4-AP). 
The platelets were diluted to $300 \times 10^{9}$ platelets/l in HEPES buffer $(137 \mathrm{mM} \mathrm{NaCl}, 2.7 \mathrm{mM}$ $\mathrm{KCl}, 1 \mathrm{mM} \mathrm{MgCl} 2,5.6 \mathrm{mM}$ glucose, $1 \mathrm{~g} / \mathrm{l}$ bovine serum albumin and 20mM HEPES, $\mathrm{pH}$ 7.40). The dilution was based on the platelet concentration on day 0 .

Spontaneous expression of GPIb was analysed as described previously [15]. Platelets were detected based on their forward and side scatter properties and assessed as percentage of GPIb-positive platelets and median fluorescence intensity (MFI).

Expression of P-selectin, LAMP-1 and PS was examined by diluting the platelet suspension (300 $\times 10^{9}$ platelets/l) 1:12 in HEPES buffer (supplemented with 2.5mM calcium, as calcium is needed for PS evaluation [18, 19] containing antibodies and, when indicated, platelet agonists. Samples were incubated for $10 \mathrm{~min}$ in room temperature, diluted 1:20 in HEPES buffer (with calcium) and analysed by flow cytometry.

Acquisition on the flow cytometer was done in Enhanced Wide angle mode (W2) for forward scatter (FSC) to allow detection and separation of smaller particles [20], with the threshold set on PE-fluorescence (corresponding to the GPIIb-antibody), as this allows detection of small platelet-derived particles that would otherwise be removed by a FSC threshold. As GPIIb/IIIa is the most abundant platelet-specific antigen (80,000 copies/platelet [21]), fluorescent triggering from this receptor allowed reliable detection of small platelet-derived particles. Further, each antibody preparation was confirmed not to cause any signal on its own in buffer without cells (data not shown), as this might be a problem during detection of microparticles [20]. At least 7,500 platelets were detected in each sample, using a temporary platelet gate. During data analysis, gates were set dividing platelet-derived particles into subpopulations (normal-sized platelets, small platelets and platelet fragments). These gates were based on 
anti-GPIIb-PE fluorescence and FSC properties and placed after visual inspection of resting (spontaneous expression) and activated samples (Fig. 1). P-selectin, PS and LAMP-1 exposure are presented as as MFI and percentage of positive platelets, where gates in the negative control samples were set to $1-2 \%$ positive platelets [22]. Data analysis was performed using Kaluza v.1.3 (Beckman Coulter).

\section{Statistics}

Results are presented as mean \pm standard deviation (SD). Comparisons between two groups were evaluated using paired t-test. Comparisons between three or more groups were evaluated by repeated measures ANOVA with Bonferroni's multiple comparisons test using GraphPad Prism (v5.02, GraphPad software Inc., San Diego, CA, USA). A p-value of $<0.05$ was considered significant. As the statistical method does not allow series with missing values, only complete series could be included, i.e. $n=4-5$ for statistical analysis comparing differences between days. However, all available data points are presented in the figures, except for the day 12 results of the PC with reduced swirling. The results from this PC are presented separately and analysed together with the other PC with reduced swirling grade. 


\section{Results}

\section{PC characteristics during storage}

All PCs (n=6) had well maintained (+++) swirling until day 7, but on day 12, one PC showed reduced swirling (+). The glucose consumption rates between day 1-7 and day 7-12 were $0.064 \pm 0.009$ and $0.074 \pm 0.005 \mathrm{mmol} / 10^{11}$ platelets/day, respectively $(P<0.05)$. The lactate formation rates between day $1-7$ and day $7-12$ were $0.124 \pm 0.006$ and $0.143 \pm 0.027 \mathrm{mmol} / 10^{11}$ platelets/day, respectively $(P<0.05)$. The unit with minimal swirling on day 12 had a $\mathrm{pH}$ below the acceptable level of 6.4. This unit had the lowest glucose level in the beginning of the study and it was depleted by day 12. $\mathrm{pH}$ remained $>6.4$ throughout storage for all other PCs (Table 1).

\section{Expression of GPIb, P-selectin, phosphatidylserine and LAMP-1 on all platelets}

The spontaneous expression of GPIb remained high throughout storage as percentage positive cells $(95 \pm 1 \%$ on day 12$)$ and MFI ( $34 \pm 6$ day 1 and $32 \pm 10$ day $12, \mathrm{P}>0.05)$ in all units except on day 12 in the unit with reduced swirling which had an expression of $88 \%$ and MFI of 16. Spontaneous expression of P-selectin increased significantly with time as did PS exposure but PS was only significantly higher on day 12. Spontaneous expression of LAMP-1 was unchanged during storage (Fig. 2). The unit with reduced swirling on day 12 had higher expression of P-selectin (96\%, MFI 30) and PS (28\%, MFI 0.3) than the other units but did not differ in LAMP-1 expression (11\%, MFI 1.1).

Agonist-induced expression of PS and LAMP-1 (after subtraction of the spontaneous expression at the same time point) is presented in Fig. 3 and S1. Only the combinations with CRP-XL and PAR-APs increased the expression of PS as compared to spontaneous expression, but the increase was clearly reduced from day 5 (Fig. 3A and S1A). LAMP-1 expression increased after stimulation with all agonists. The increase was gradually reduced 
throughout the storage period but was not significantly lower until day 12 (Fig. 3B and S1B). The PC with reduced swirling on day 12 showed no increase in LAMP-1 expression with activation $(\leq 0.6 \%)$, while agonist-induced PS exposure was still present $(8.9-21.5 \%$ for the different agonists).

\section{Formation of platelet subpopulations}

Storage for 7 days did not induce any significant spontaneous formation of small platelets and platelet fragments, i.e. platelets remained normal-sized (Fig. 4A). By day 12 a significant fraction of platelets had become small, but these still made up less than 5\% of all plateletderived particles (Fig. 4B).

Small platelets were mainly formed when platelets were activated via the collagen receptor GPVI-FcR $\gamma$ with CRP-XL. This was associated with the appearance of another population of substantially smaller platelet-derived particles, referred to as "platelet fragments" (Fig. 4C and 1). The production of small platelets and platelet fragments increased substantially when CRP-XL was combined with PAR-APs to involve the thrombin receptors PAR1 and PAR4, whereas PAR-APs alone showed low potency. The combination of CRP-XL+ PAR-APs caused a significant formation of small platelets and fragments on day 1 , but this was drastically reduced already on day 5 for all CRP-XL+PAR-AP combinations, with a further decrease on day 7 for the combination of all three agonists (Fig. 4B and C). The PC with reduced swirling on day 12 could not form platelet subpopulations (Fig. 6B).

\section{Expression of phosphatidylserine and LAMP-1 on platelet subpopulations after agonist}

\section{stimulation}

As only the combination of CRP-XL with PAR-APs caused a significant formation of platelet subpopulations, we only assessed the expression of PS and LAMP-1 on the three platelet subpopulations after stimulation with these combinations. Expression of PS following agonist 
stimulation was initially high on small platelets and fragments, while the normal-sized platelets expressed relatively low levels, which was also reflected in the results for all platelet-derived particles (Fig. 5A-C and S2A-C). The expression decreased on all plateletderived particles, small platelets and fragments during storage whereas the PS response in normal-sized platelets was unaltered. In contrast, expression of LAMP-1 after agonist stimulation was initially high on normal-sized, small platelets and all platelet-derived particles. The expression decreased gradually and was significantly lower on day 12 (Fig. 5DF and S2D-F).

\section{Comparison of PCs with different swirling grade}

As one PC showed reduced swirling on day 12, this PC was compared to 5 other PCs stored until their swirling decreased. Four PCs were without swirling, whereas one showed reduced swirling. These PCs did not form subpopulations upon agonist stimulation (Fig. 6). Therefore expression of PS and LAMP-1 was evaluated on all platelet-derived particles.

Comparison of PCs with different swirling grade revealed that the spontaneous expression of P-selectin and PS increased as the swirling ability decreased (Fig. 7A), but for P-selectin, some of the PCs with retained swirling had an expression similar to the ones without swirling (Fig. 7B). Furthermore, PCs with retained swirling were able to express LAMP-1 in response to agonist stimulation, although to a varying degree, while no agonist-induced LAMP-1 expression was seen in PCs with reduced or without swirling (Fig. 7C-D). 


\section{Discussion}

This study investigated the activation capacity of platelets from PCs during extended storage for 12 days and their ability to respond to activation by formation of subpopulations. As swirling is a quality indicator used routinely, further comparisons were made between PCs with fully retained swirling and PCs with reduced or lost swirling. Our results showed that stimulation via the collagen receptor GPVI-FcR $\gamma$ played a major role in inducing formation of small platelets and platelet fragments, as these were only observed when CRP-XL was used as a platelet activator. The production was however substantially potentiated when PAR-APs were added as well. With this flow cytometer and the fluorescence trigger approach, we found that platelets not only formed the normally reported, normal-sized platelets and "microparticles", but actually formed three subpopulations. Our findings are supported by other reports questioning previous gating strategies as they found that the numbers of reported "microparticles" formed in vitro were too low, suggesting that the detected particles were more likely "small platelets" [23]. The small platelets and platelet fragments presumably stem from the same platelets, as both of these subpopulations showed high PS exposure. Since PS exposure is a prerequisite for binding of coagulation factors, these platelets can be considered "procoagulant". The platelet subpopulation that kept the normal size had a relatively low PS exposure, but we and others have reported that these platelets instead express an activated fibrinogen receptor and thus would be the ones able to participate in aggregation [24-26]. One interesting finding in this study was that the capacity of platelets to undergo activationinduced subpopulation formation was drastically decreased with storage, with significant changes already on day 5 for some platelet agonists, even in this limited material. The PS exposure by small platelets and platelet fragments upon activation also decreased with time, while the normal-sized platelets showed no clear trend. One reason for this might be that the spontaneous PS exposure increased with time in this population, another that fragmentation 
was the property most severely affected by storage, and that some platelets presumably turned PS positive without fragmenting in the older samples.

For the lysosomal exocytosis capacity, measured as LAMP-1 expression, the trend was different from the PS exposure, as the normal-sized and small platelets showed the highest expression upon activation. Agonist-induced LAMP-1 expression decreased gradually with storage for all subpopulations, while the spontaneous expression was relatively unaltered. Significant changes with storage were seen for platelets activated with all agonists tested, and as this parameter is not obscured by the spontaneous increase with storage normally reported for PS [19, 22, 27, 28] and P-selectin exposure [15, 27, 29], LAMP-1 could be an interesting marker to follow changes in activation-induced responses of stored platelets.

The reduced swirling on day 12 in one PC was likely explained by glucose depletion since glucose depletion can result in poor $\mathrm{pH}$ which affects swirling and in vivo viability $[16,30]$. We felt it was interesting to further investigate how changes in swirling was reflected in our platelet function analyses. Therefore, we stored some additional PCs until their swirling became affected. The most striking finding was that platelets from PCs with reduced or lost swirling were no longer able to form platelet subpopulations and that the agonist-induced LAMP-1 expression was completely abolished. PCs with reduced or lost swirling also showed an increase in PS expression but as these differences were most obvious upon comparison of different PCs, this marker may be more difficult to evaluate in single PCs. Loss of swirling and a low $\mathrm{pH}$ are good predictors of poor PC quality. These parameters may however be too insensitive to detect more subtle changes in PC function [31]. Interestingly, the agonistinduced LAMP-1 expression showed a relatively large variation among PCs with retained swirling. If further studies can confirm that PCs with lower responses are closer to becoming non-responsive, this could be a useful marker to identify PCs where platelet function is 
starting to decline before changes in swirling can be seen. It might also provide a more objective analysis of function than visually assessing the swirling grades.

Previous studies agree that platelets prepared by apheresis lose their function during storage as evident by a gradual reduction in activation response to TRAP-6 (PAR1-AP) detected by flow cytometry $[15,27]$ and reduced aggregation response to collagen [32] but to a various degree depending on apheresis equipment [32]. Platelet storage also reduces the in vivo recovery and survival [33] but so far, relatively few studies have investigated the correlation between in vitro variables and in vivo viability. In general, the correlations reported have been rather low and varying between studies [31, 33-35], something likely reflecting the difficulty in measuring in vivo viability in heterogeneous patient populations [34]. New in vitro test strategies such as those investigating the platelet activation potential could potentially contribute to better results in this regard. From this study and previously published data, we suggest that agonist-induced platelet responses should be evaluated in studies of stored PCs such as assessing new preparation methods. In addition to P-selectin exposure, we propose that a basic protocol includes the formation of "platelet-derived particles smaller than resting platelets" (here corresponding to small platelets and platelet fragments) and expression of LAMP-1 and PS on "all platelet-derived particles" in resting platelets (spontaneous expression) and in response to a combined stimuli including CRP-XL and at least one of the PAR-APs. This approach should enable studies to be performed also with less advanced flow cytometers. Compared to e.g. changes in receptor levels such as GPIb, where both unaltered $[15,36]$ and decreased levels $[28,37]$ have been reported, we believe that our parameters are more sensitive and therefore more likely to pick up subtle changes in platelet functionality during the early phases of storage. The suggested protocol now needs to be evaluated in larger studies, also allowing subgroup analysis of different apheresis modalities and different kinds of PCs (e.g. buffy coat concentrates), as this was not possible in the present study. Hopefully 
this protocol may contribute to better estimations of platelet functionality and increased knowledge regarding factors important for successful and effective platelet transfusion therapy.

\section{Acknowledgements}

Data acquisition and analysis was performed by ALS and NT. All authors took part in experimental design, interpretation of data and preparation of the manuscript. We thank our collaborators at the Department of Clinical Chemistry and Clinical Immunology and Transfusion Medicine at Linköping University Hospital for their assistance. This study was supported by grants from Region Östergötland and Linköping University through the LiU Research Fellows program.

\section{Declaration of Interests}

The authors report no conflicts of interests.

\section{References}

1. Sims PJ, Wiedmer T, Esmon CT, et al.: Assembly of the platelet prothrombinase complex is linked to vesiculation of the platelet plasma membrane. Studies in scott syndrome: An isolated defect in platelet procoagulant activity. J Biol Chem 1989; 264:17049-17057. 2. Heemskerk JW, Mattheij NJ, Cosemans JM: Platelet-based coagulation: Different populations, different functions. J Thromb Haemost 2013; 11:2-16.

3. Mazepa M, Hoffman M, Monroe D: Superactivated platelets: Thrombus regulators, thrombin generators, and potential clinical targets. Arterioscler Thromb Vasc Biol 2013; 33:1747-1752. 
4. Cauwenberghs S, van Pampus E, Curvers J, et al:: Hemostatic and signaling functions of transfused platelets. Transfus Med Rev 2007; 21:287-294.

5. Ohto H, Nollet KE: Overview on platelet preservation: Better controls over storage lesion. Transfus Apher Sci 2011; 44:321-325.

6. Cardigan R, Turner C, Harrison P: Current methods of assessing platelet function:

Relevance to transfusion medicine. Vox Sang 2005; 88:153-163.

7. Panzer S, Jilma P: Methods for testing platelet function for transfusion medicine. Vox Sang 2011; 101:1-9.

8. Tynngard N: Preparation, storage and quality control of platelet concentrates. Transfus Apher Sci 2009; 41:97-104.

9. Jilma-Stohlawetz P, Eichelberger B, Horvath M, et al.: In vitro platelet function of platelet concentrates prepared using three different apheresis devices determined by impedance and optical aggregometry. Transfusion 2009; 49:1564-1568.

10. Bochsen L, Johansson PI, Kristensen AT, et al.: The influence of platelets, plasma and red blood cells on functional haemostatic assays. Blood Coagul Fibrinolysis 2011; 22:167175.

11. Xu Y, Nakane N, Maurer-Spurej E: Novel test for microparticles in platelet-rich plasma and platelet concentrates using dynamic light scattering. Transfusion 2011; 51:363-370.

12. Febbraio M, Silverstein RL: Identification and characterization of lamp-1 as an activation-dependent platelet surface glycoprotein. J Biol Chem 1990; 265:18531-18537. 13. Ciferri S, Emiliani C, Guglielmini G, et al:: Platelets release their lysosomal content in vivo in humans upon activation. Thromb Haemost 2000; 83:157-164.

14. Emiliani C, Ciferri S, Mencarelli S, et al.: Defective platelet beta-n-acetyl hexosaminidase content and release in chronic myeloproliferative disorders. Platelets 2006; 17:20-29. 
15. Tynngård N, Lindahl TL, Trinks M, et al:: The quality of platelet concentrates produced by cobe spectra and trima accel cell separators during storage for 7 days as assessed by in vitro methods. Transfusion 2008; 48:715-722.

16. Bertolini F, Murphy S: A multicenter inspection of the swirling phenomenon in platelet concentrates prepared in routine practice. Biomedical excellence for safer transfusion (best) working party of the international society of blood transfusion. Transfusion 1996; 36:128132.

17. Morton LF, Hargreaves PG, Farndale RW, et al.: Integrin alpha 2 beta 1 -independent activation of platelets by simple collagen-like peptides: Collagen tertiary (triple-helical) and quaternary (polymeric) structures are sufficient alone for alpha 2 beta 1-independent platelet reactivity. Biochem J 1995; 306:337-344.

18. Andree HA, Reutelingsperger CP, Hauptmann R, et al.: Binding of vascular anticoagulant alpha (vac alpha) to planar phospholipid bilayers. J Biol Chem 1990; 265:49234928.

19. Albanyan AM, Harrison P, Murphy MF: Markers of platelet activation and apoptosis during storage of apheresis- and buffy coat-derived platelet concentrates for 7 days. Transfusion 2009; 49:108-117.

20. Lacroix R, Robert S, Poncelet P, et al:: Overcoming limitations of microparticle measurement by flow cytometry. Semin Thromb Hemost 2010; 36:807-818.

21. Wagner CL, Mascelli MA, Neblock DS, et al.: Analysis of gpiib/iiia receptor number by quantification of 7e3 binding to human platelets. Blood 1996; 88:907-914.

22. Saunders C, Rowe G, Wilkins K, et al.: In vitro storage characteristics of platelet concentrates suspended in $70 \% \mathrm{ssp}+(\mathrm{tm})$ additive solution versus plasma over a 14-day storage period. Vox Sang 2011; 101:112-121. 
23. Bode AP, Hickerson DH: Characterization and quantitation by flow cytometry of membranous microparticles formed during activation of platelet suspensions with ionophore or thrombin. Platelets 2000; 11:259-271.

24. Munnix IC, Kuijpers MJ, Auger J, et al.: Segregation of platelet aggregatory and procoagulant microdomains in thrombus formation: Regulation by transient integrin activation. Arterioscler Thromb Vasc Biol 2007; 27:2484-2490.

25. Södergren A, Öllinger K, Ramström S: Subdivision according to size is necessary for correct interpretation of data regarding platelet expression of active gpiib/iiia and phosphatidylserine. J Thromb Haemost 2013; 11 Suppl 2:498.

26. Topalov NN, Yakimenko AO, Canault M, et al.: Two types of procoagulant platelets are formed upon physiological activation and are controlled by integrin alpha(iib)beta(3). Arterioscler Thromb Vasc Biol 2012; 32:2475-2483.

27. Curvers J, van Pampus EC, Feijge MA, et al.: Decreased responsiveness and development of activation markers of plts stored in plasma. Transfusion 2004; 44:49-58. 28. Cookson P, Sutherland J, Turner C, et al:: Platelet apoptosis and activation in platelet concentrates stored for up to 12 days in plasma or additive solution. Transfus Med 2010; 20:392-402.

29. Zhang JG, Carter CJ, Culibrk B, et al.: Buffy-coat platelet variables and metabolism during storage in additive solutions or plasma. Transfusion 2008; 48:847-856.

30. Murphy S: Platelet storage for transfusion. Semin Hematol 1985; 22:165-177.

31. Rinder HM, Smith BR: In vitro evaluation of stored platelets: Is there hope for predicting posttransfusion platelet survival and function? Transfusion 2003; 43:2-6.

32. Macher S, Sipurzynski-Budrass S, Rosskopf K, et al.: Function and activation state of platelets in vitro depend on apheresis modality. Vox Sang 2010; 99:332-340. 
33. Slichter SJ, Bolgiano D, Corson J, et al.: Extended storage of autologous apheresis platelets in plasma. Vox Sang 2013; 104:324-330.

34. Holme S: Storage and quality assessment of platelets. Vox Sang 1998; 74 Suppl 2:207216.

35. Cardigan R, Williamson LM: The quality of platelets after storage for 7 days. Transfus Med 2003; 13:173-187.

36. Albanyan AM, Murphy MF, Rasmussen JT, et al.: Measurement of phosphatidylserine exposure during storage of platelet concentrates using the novel probe lactadherin: A comparison study with annexin v. Transfusion 2009; 49:99-107.

37. Sandgren P, Hansson M, Gulliksson H, et al:: Storage of buffy-coat-derived platelets in additive solutions at 4 degrees c and 22 degrees c: Flow cytometry analysis of platelet glycoprotein expression. Vox Sang 2007; 93:27-36. 


\section{Figure \& table legends}

\section{Table 1.}

Results are presented as mean \pm SD for $n=6$ except on Day 12 where $n=5$. Results for the PC with reduced swirling on day 12 are shown within parentheses. $*=P<0.05$ and $* *=P<0.01$ as compared to day 1 . \#\# $=P<0.01$ for day 7 vs. day 12 . PLT-platelet, MPV-mean platelet volume, $\mathrm{LDH}-$ lactate dehydrogenase, $(1 \mu \mathrm{kat} / \mathrm{l}=60 \mathrm{U} / \mathrm{l})$.

Fig. 1 Gating strategy for platelet subpopulations.

Placement of gates dividing all platelet-derived particles (All plt) into platelet subpopulations; Normal-sized platelets (light grey), small platelets (black) and platelet fragments (dark grey). Dot plots shown are from one representative PC, on day 1, and shows (A) a resting sample, i.e. without agonist activation and (B) the appearance of platelet subpopulations after stimulation with CRP-XL+PAR1-AP+PAR4-AP. Forward scatter (FSC) corresponds to platelet size and GPIIb-PE represents the fluorescence signal from the platelet specific GPIIbPE-antibody. Plt-platelet, CRP-XL- cross-linked collagen-related peptide, PAR1/4-APPAR1/4-activating peptide.

Fig. 2 Spontaneous expression of GPIb (A), P-selectin (B), PS (C) and LAMP-1 (D) on all platelet-derived particles during storage. Filled bars show \% positive platelets, while coarse bars show median fluorescence intensity (MFI). Results are presented as mean \pm SD for $n=6$ except on day 5 and 12 where $n=5$. $*=P<0.05$ and $* *=P<0.01$ compared to day 1 and \# $=P<0.05$ for day 7 vs. day 12 . PS-phosphatidylserine, LAMP-1-lysosome associated membrane protein-1. 
Fig. 3 Agonist-induced expression of PS and LAMP-1 on all platelet-derived particles. Agonist-induced expression of (A) PS and (B) LAMP-1, presented as percentage of positive platelets after subtraction of the spontaneous expression at the same time point. Results are presented as mean $\pm \mathrm{SD}$ for $\mathrm{n}=6$ except on day 5 and 12 where $\mathrm{n}=5 . *=\mathrm{P}<0.05$ and $* *=\mathrm{P}<0.01$ compared to day 1 . \# =P<0.05 and \#\# =P<0.01, for day 7 vs. day 12 . PS-phosphatidylserine, LAMP-1-lysosome associated membrane protein-1, CRP-XL-cross-linked collagen related peptide, PAR1/4-AP-PAR1/4-activating peptide.

Fig. 4 Changes in the formation of platelet subpopulations in resting and activated samples. Figures shows the fraction (\%) of all platelet-derived particles that are (A) normal-sized platelets, (B) small platelets and (C) platelet fragments. Results are presented as mean \pm SD for $\mathrm{n}=6$ except on day 5 and 12 where $\mathrm{n}=5 . *=P<0.05$ and $* *=P<0.01$ compared to day $1 .+$ $=P<0.05$ and $++=P<0.01$ compared to the respective resting sample. $\mathrm{CRP}-\mathrm{XL}-$ cross-linked collagen related peptide, PAR1/4-AP-PAR1/4-activating peptide.

Fig. 5 Expression of PS and LAMP-1 on platelet subpopulations after agonist stimulation. The fraction (\%) of PS and LAMP-1 positive platelets on all platelets and in the different platelet subpopulations on day 1, 5, 7 and 12 of storage after stimulation with combinations of agonists; (A, D) CRP-XL+PAR1-AP, (B, E) CRP-XL+PAR4-AP, and (C, F) CRP$\mathrm{XL}+\mathrm{PAR} 1-\mathrm{AP}+\mathrm{PAR} 4-\mathrm{AP}$. Results are presented as mean $\pm \mathrm{SD}$ for $\mathrm{n}=6$ except on day 5 and 12 where $\mathrm{n}=5 . *=\mathrm{P}<0.05$ and $* *=\mathrm{P}<0.01$ compared to day 1 . $\#=\mathrm{P}<0.05$ for day 7 vs. day 12 . PS-phosphatidylserine, LAMP-1-lysosome associated membrane protein-1, CRP-XL-crosslinked collagen related peptide, PAR1/4-AP-PAR1/4-activating peptide. 
Fig. 6 Gating of platelet subpopulations in PCs with different swirling grades.

The appearance of platelets with different swirling grade and when possible, gates dividing the platelet-derived particles into subpopulations. For each PC dot plots are shown for resting and activated (CRP-XL+PAR1-AP+PAR4-AP) samples. (A) A representative PC with fully retained swirling (on day 1). Normal-sized platelets (light grey), small platelets (black) and platelet fragments (dark grey). (B-C) PCs with reduced swirling. In B the same PC as in A is shown, but on day 12. (D) A representative PC without swirling. Forward scatter (FSC) corresponds to platelet size and GPIIb-PE represents the fluorescence signal from the platelet specific GPIIb-PE-antibody. Plt-platelet, CRP-XL-cross-linked collagen-related peptide, PAR1/4-AP-PAR1/4-activating peptide.

Fig. 7 Expression of activation markers on all platelet-derived particles from PCs with different swirling grades.

Expression of P-selectin, PS and LAMP-1 on platelets in resting samples (spontaneous expression) and in response to activation by CRP-XL+PAR1-AP+PAR4-AP. PCs were divided according to swirling grade; fully retained swirling (grade +++ , day 7-12, n=6), reduced swirling (grade + , day 12-22, $\mathrm{n}=2$ ) and without swirling (grade 0 , day 15-17, $\mathrm{n}=4$ ). (A) Platelet expression of P-selectin, PS and LAMP-1 in resting (spontaneous expression) and activated samples (striped bars). Results are presented as mean \pm SD. Correlation of the spontaneous expression of (B) P-selectin and PS and (C) PS and LAMP-1. (D) Correlation of PS and LAMP-1 expression after agonist stimulation. PS-phosphatidylserine, LAMP-1Lysosome associated membrane protein-1, CRP-XL- cross-linked collagen related peptide, PAR1/4-AP-protease activated receptor 1/4-activating peptide. 
Table 1. PC characteristics

$\begin{array}{lllll}\text { Variable } & \text { Day 1 } & \text { Day 5 } & \text { Day 7 } & \text { Day 12 } \\ \begin{array}{l}\text { PLT concentration } \\ \left(\mathrm{x} 10^{9} / \mathrm{l}\right)\end{array} & 1337 \pm 95 & 1429 \pm 100 & 1366 \pm 105 & 1318 \pm 128(1392) \\ \begin{array}{l}\text { Volume } \\ (\mathrm{ml})\end{array} & 215 \pm 12 & 200 \pm 16^{*} & 184 \pm 18^{* *} & 174 \pm 17 * * \# \text { (138) } \\ \begin{array}{l}\text { MPV } \\ (\mathrm{fl})\end{array} & 6.5 \pm 0.8 & 6.6 \pm 0.9 & 7.1 \pm 1.4 & 7.2 \pm 0.5(8.9) \\ \mathrm{pH} & 7.35 \pm 0.04 & 7.34 \pm 0.07 & 7.15 \pm 0.32 & 6.94 \pm 0.15 *(6.02) \\ \begin{array}{l}\mathrm{LDH} \\ (\mu \mathrm{kat} / \mathrm{l})\end{array} & 2.5 \pm 0.5 & 3.2 \pm 1.4 * & 2.5 \pm 0.3 & 2.9 \pm 0.5(4.9)\end{array}$



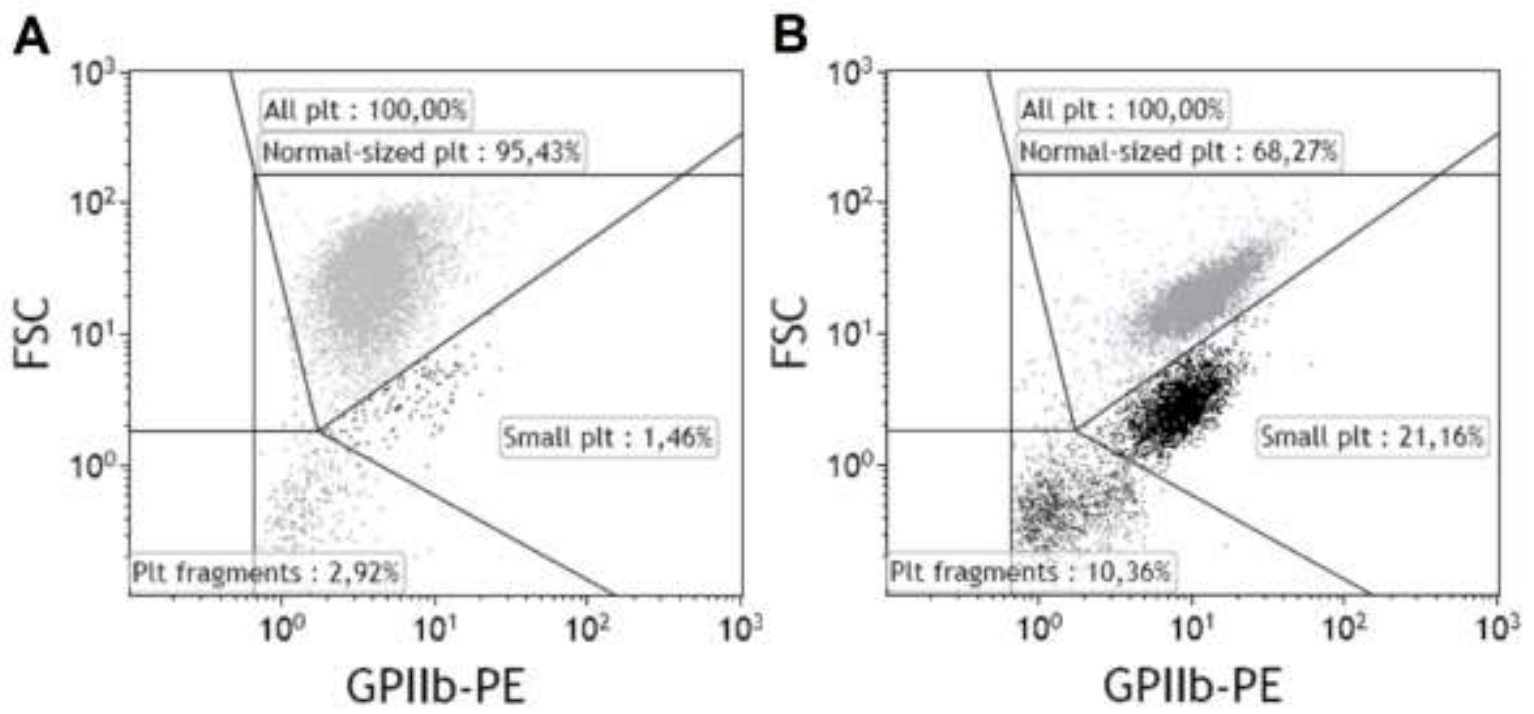

\section{Figure 1}


A

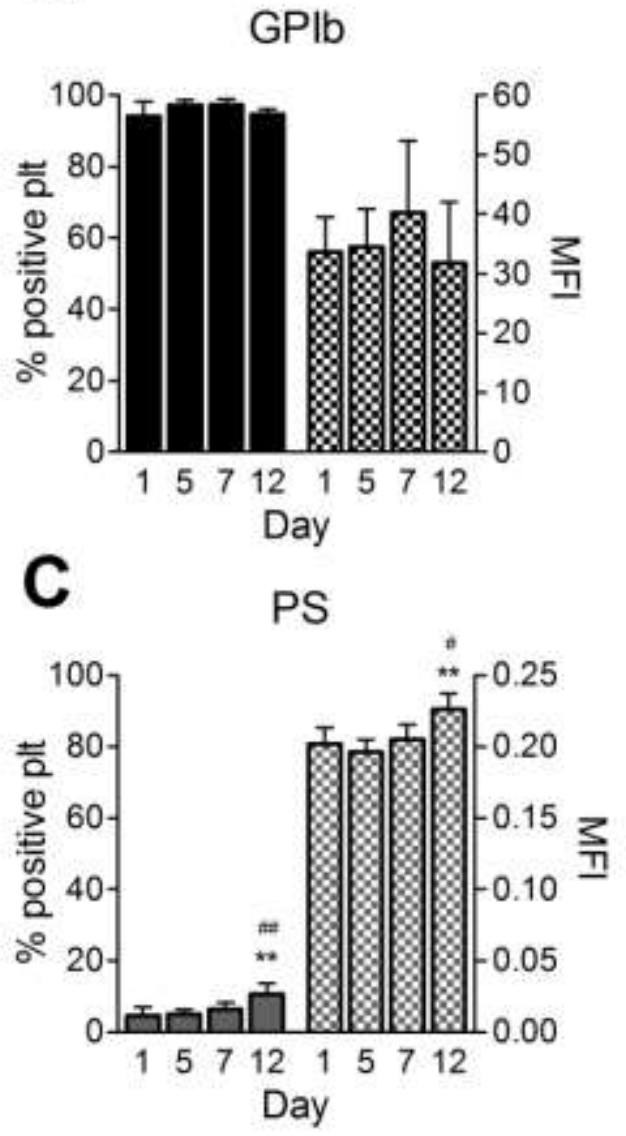

B
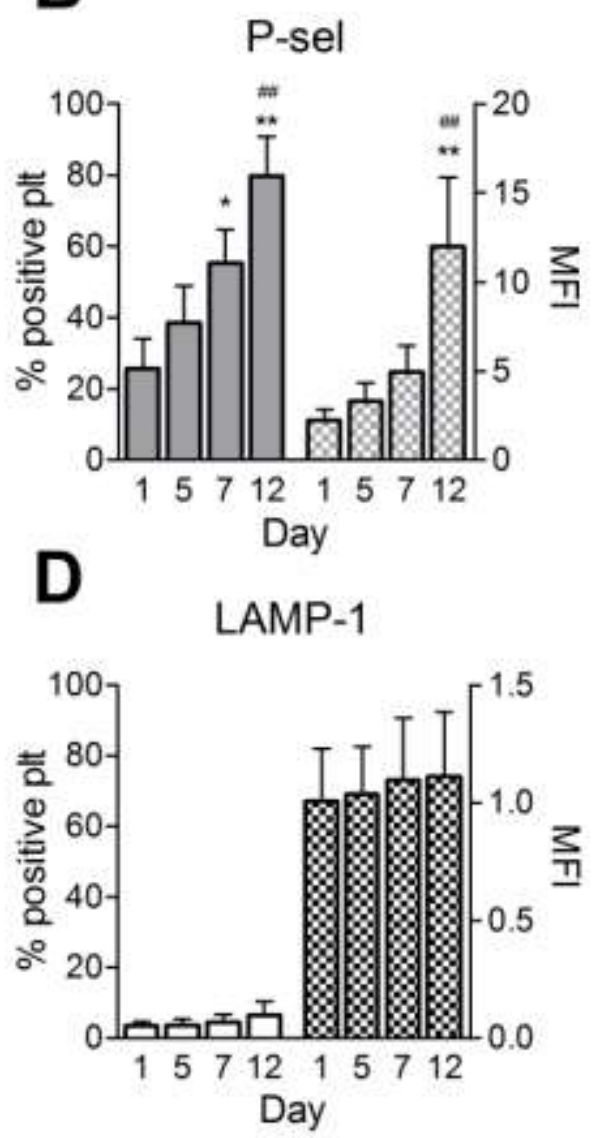

Figure 2 

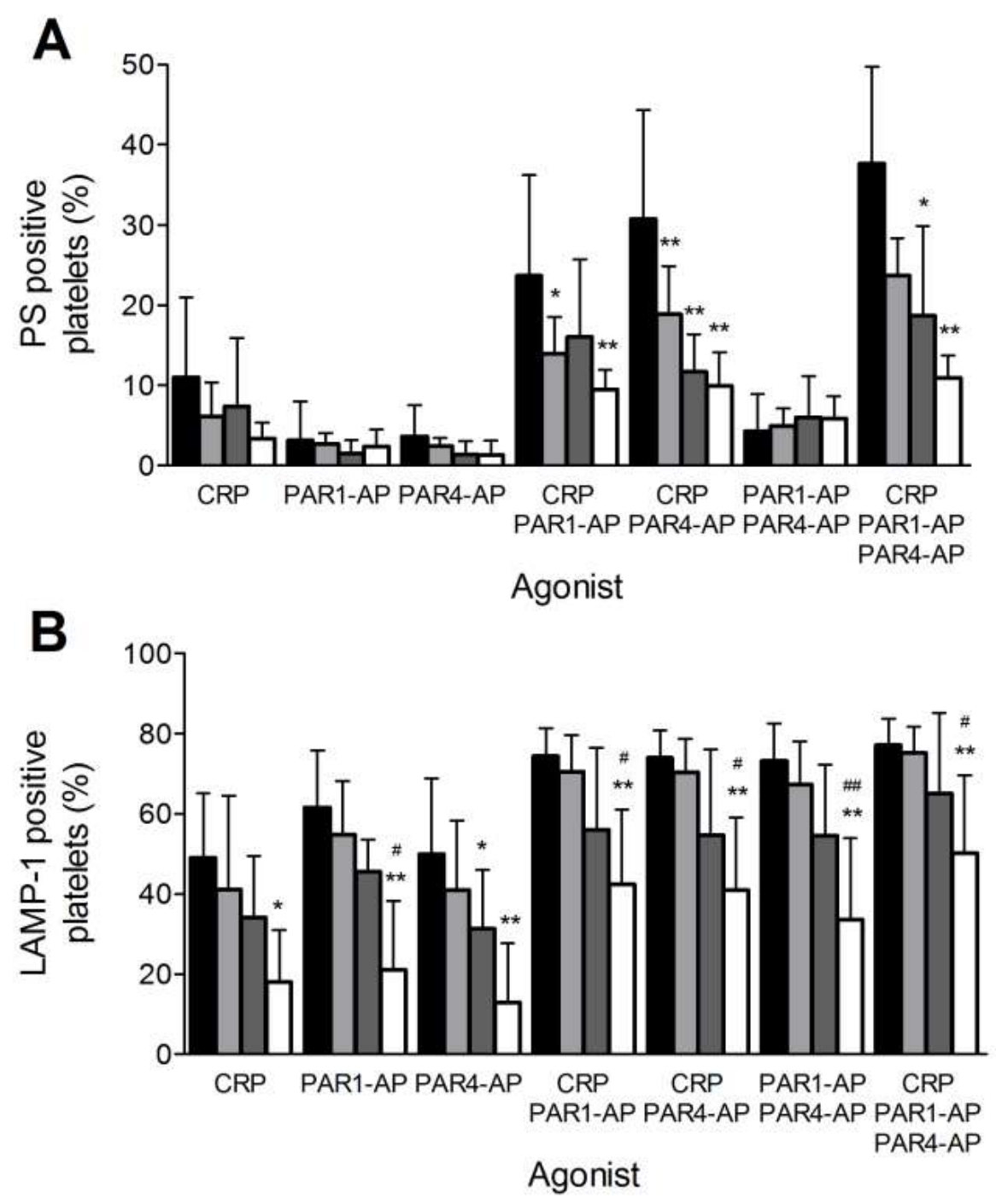

$\square$ Day $1 \square$ Day $5 \square$ Day $7 \square$ Day 12

Figure 3 


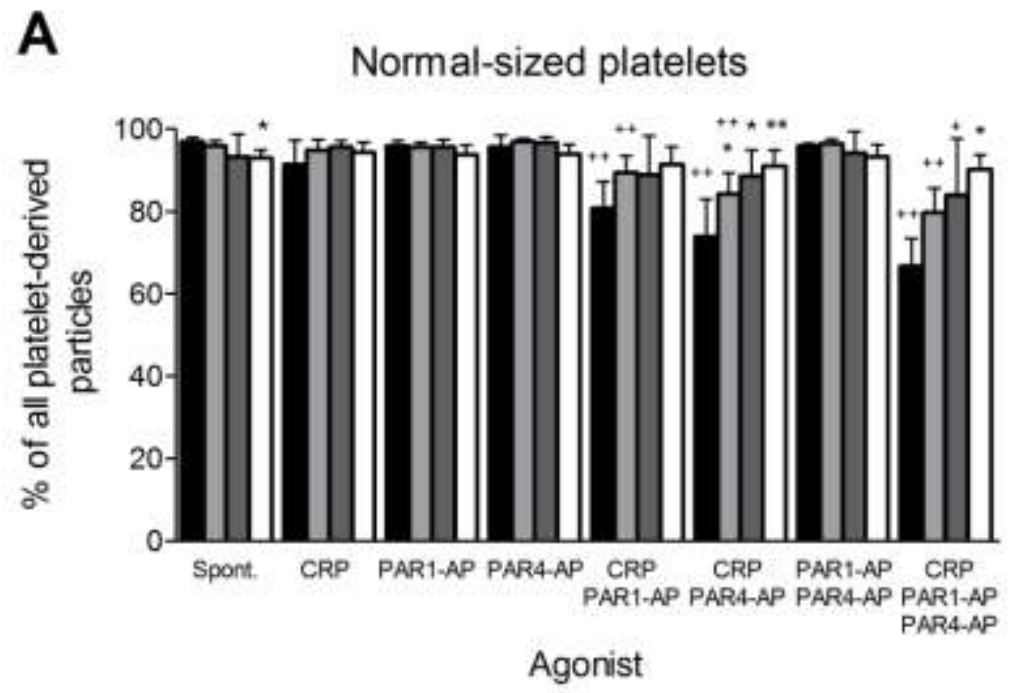

B

Small platelets

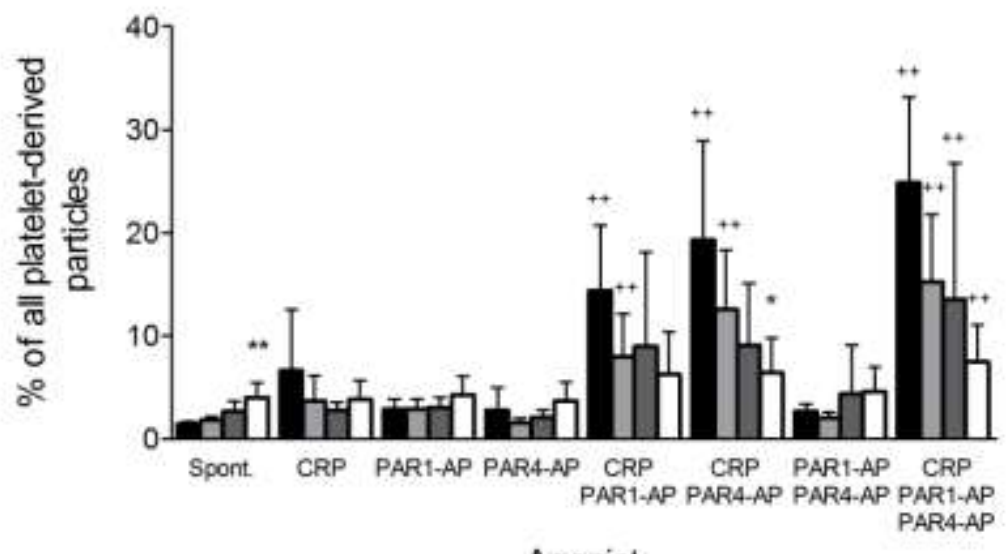

Agonist

C

Platelet fragments

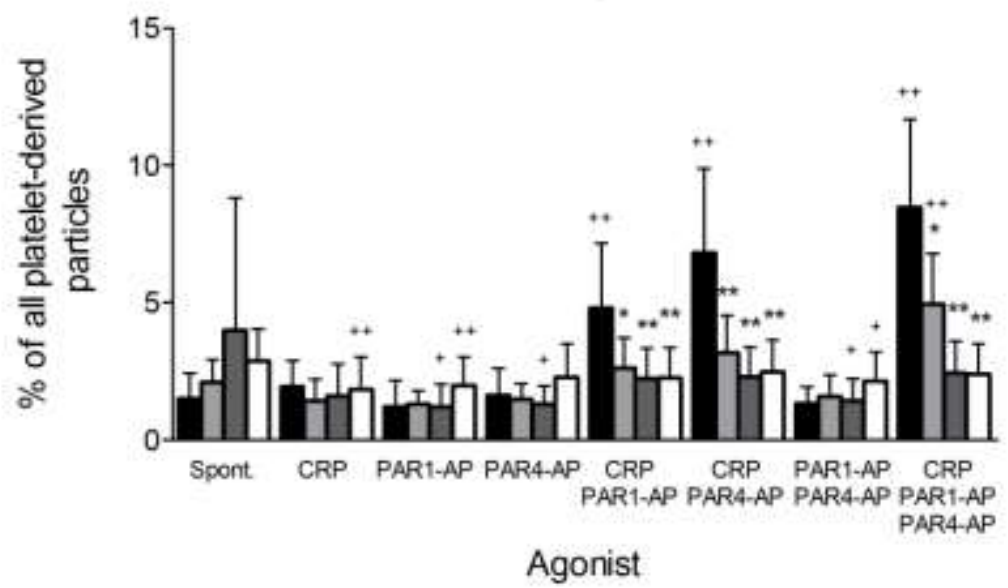

$\square$ Day $1 \square$ Day $5 \square$ Day $7 \square$ Day 12

Figure 4 
A
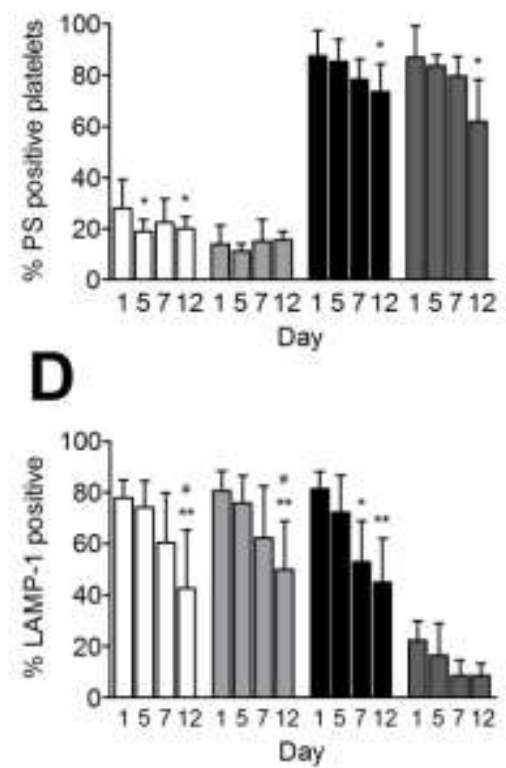

B
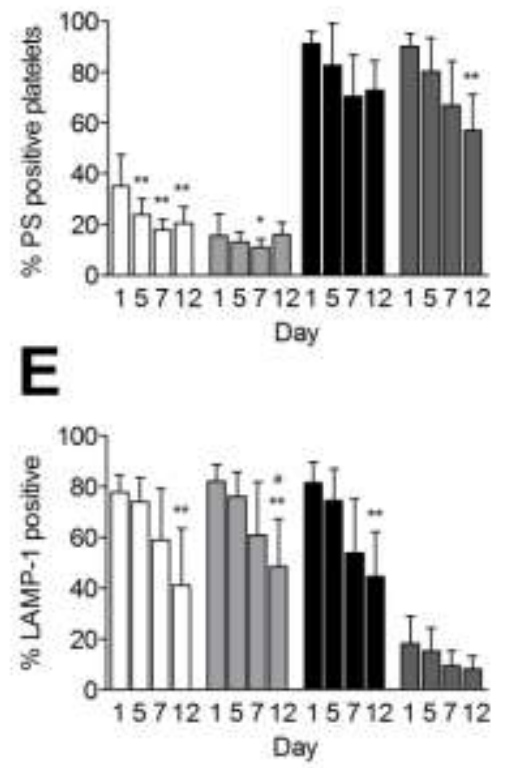

C

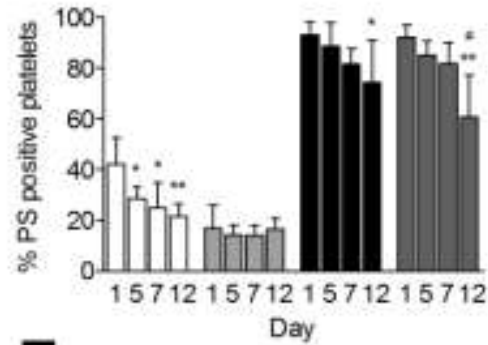

F

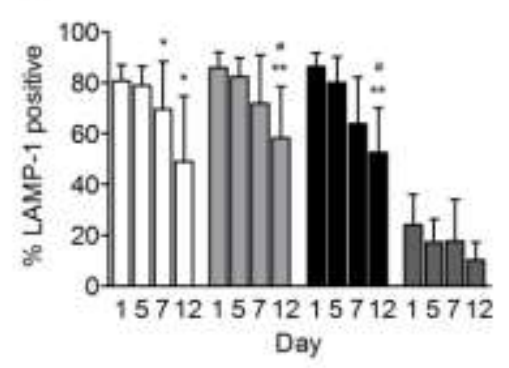

$\square$ All plt $\square$ Normal-sized plt $\square$ Small plt $\square$ Plt fragments

Figure 5 

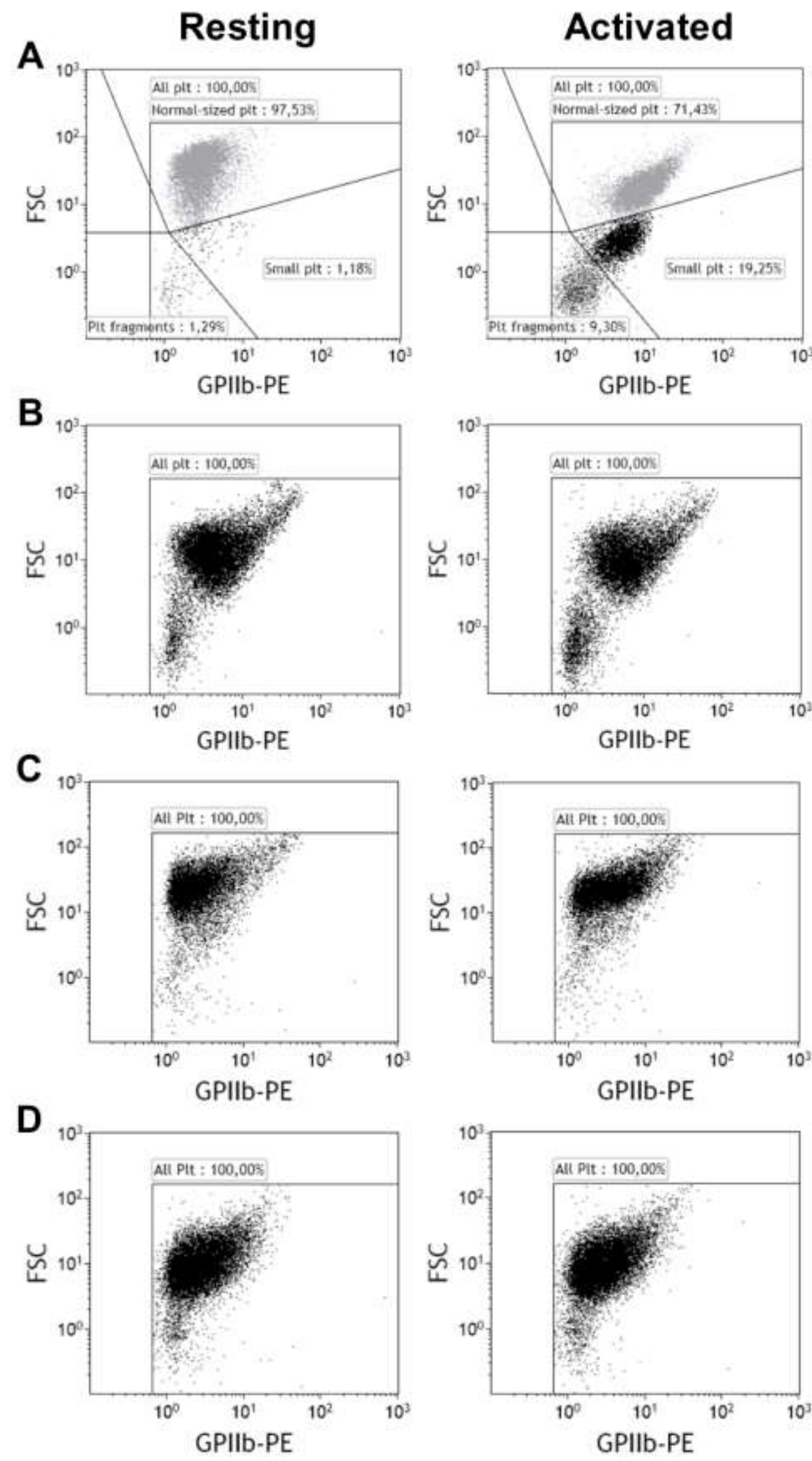

Figure 6 
A

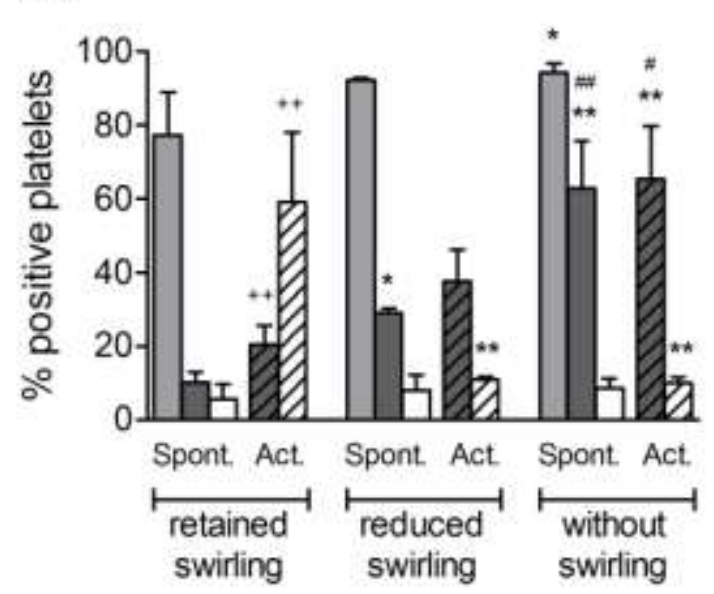

B

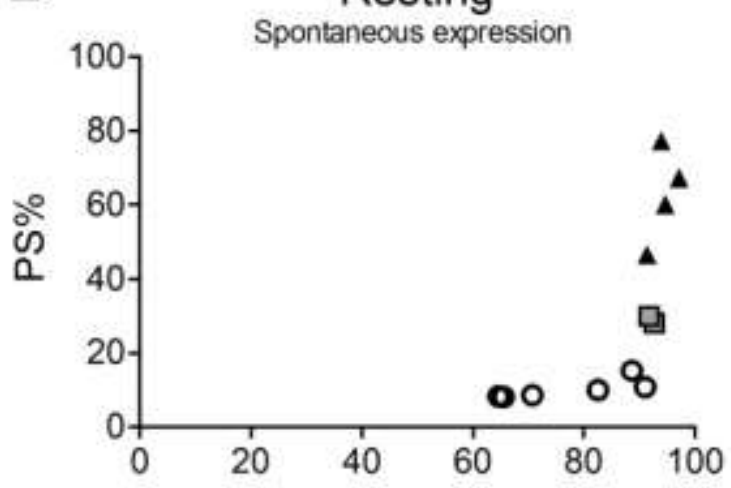

P-selectin \%
C

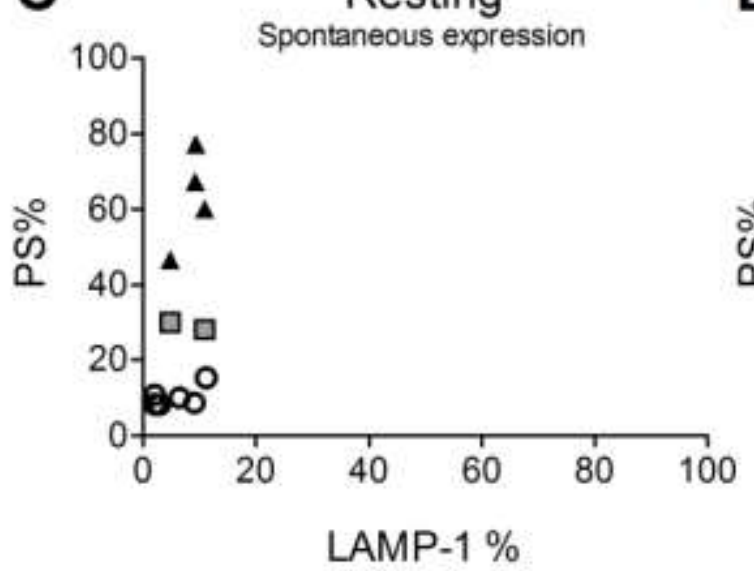

D Activated

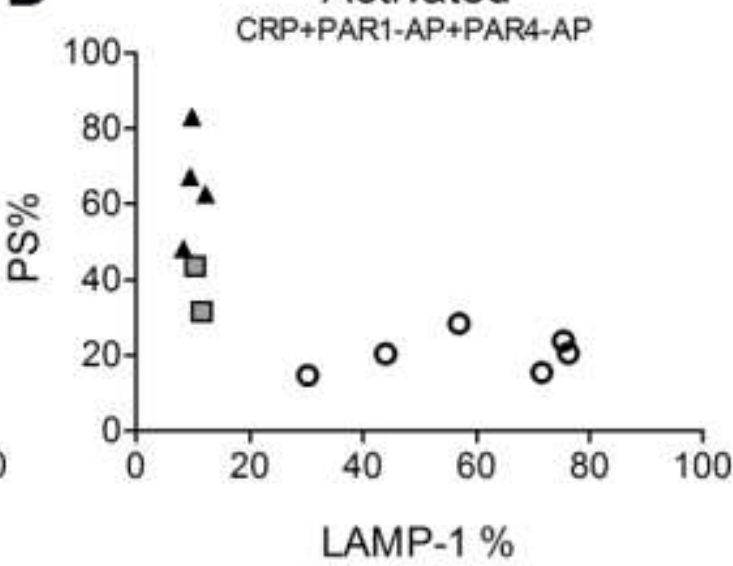

P-selectin

- Retained swirling
PS

Reduced swirling
LAMP-1

A Without swirling

Figure 7 

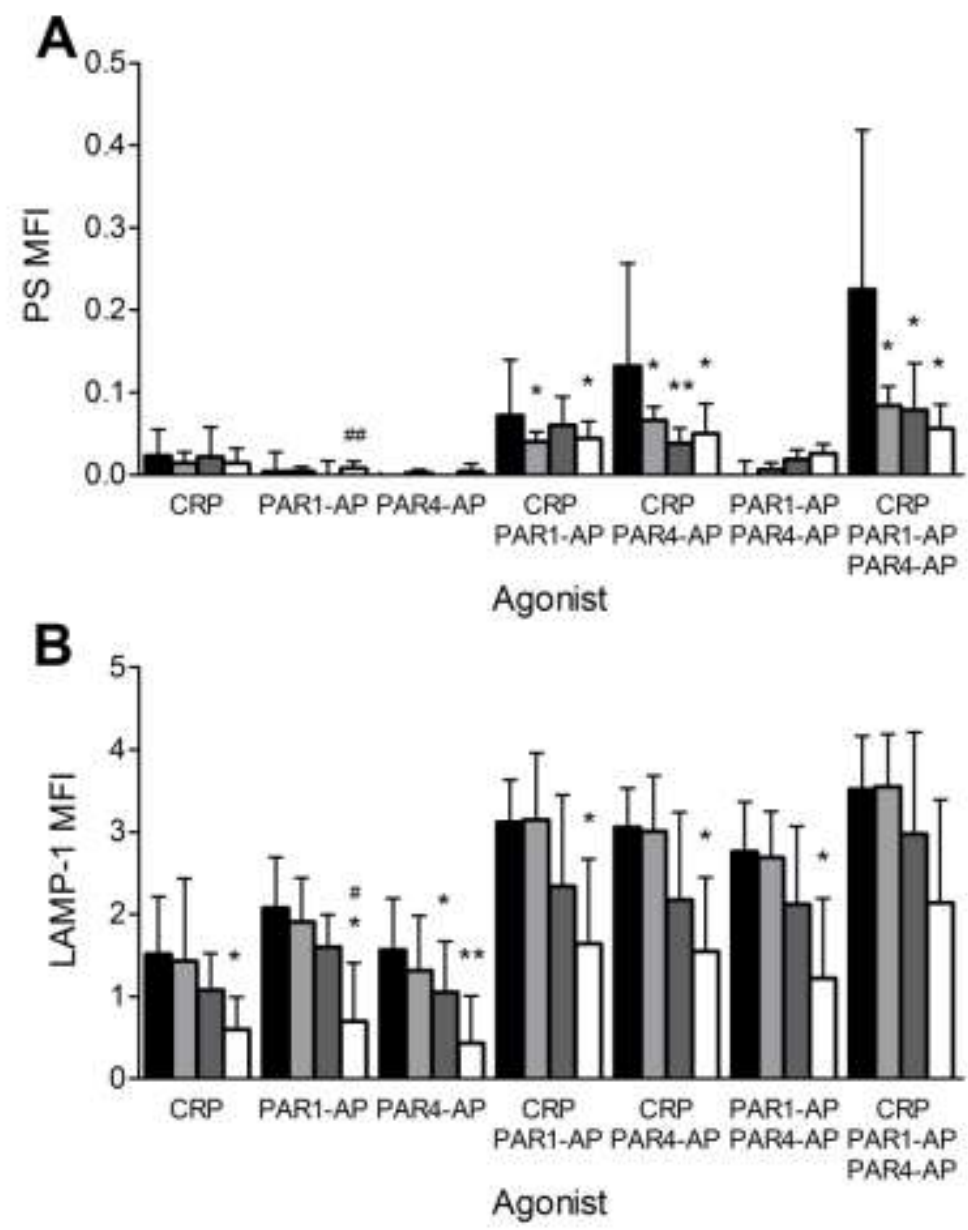

$\square$ Day $1 \square$ Day $5 \square$ Day $7 \square$ Day 12

\section{Supplementary figure S1.}

\section{Agonist-induced expression of PS and LAMP-1 on all platelet-derived particles.}

Agonist-induced expression of (A) PS and (B) LAMP-1, presented as median fluorescence intensity (MFI) of platelets after subtraction of the spontaneous expression at the same time point. Results are presented as mean $\pm \mathrm{SD}$ for $\mathrm{n}=6$ except on day 5 and 12 where $\mathrm{n}=5$. $*=\mathrm{P}<0.05$ and $* *=\mathrm{P}<0.01$ compared to day 1 . \# $=\mathrm{P}<0.05$ and $\# \#=\mathrm{P}<0.01$, for day 7 vs. day 12 . PSphosphatidylserine, LAMP-1-lysosome associated membrane protein-1, CRP-XL-crosslinked collagen related peptide, PAR1/4-AP-PAR1/4-activating peptide. 
A
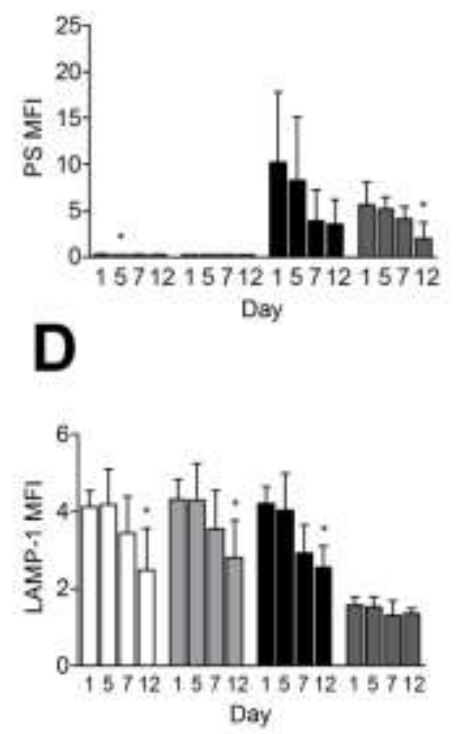

$\square$ All plt
B
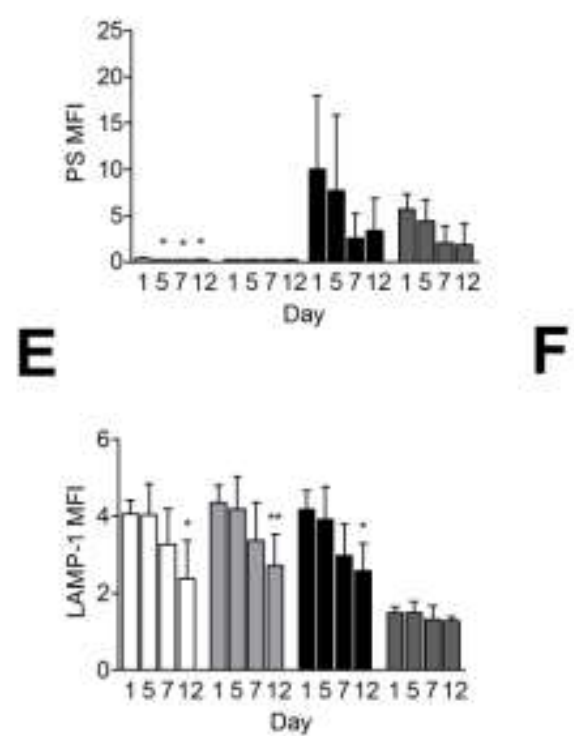

Day

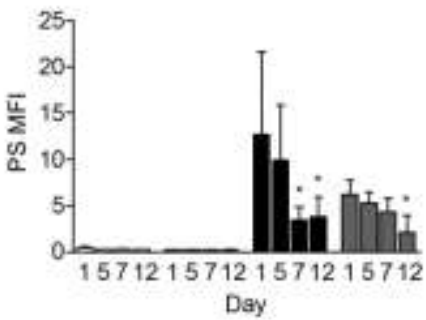

$\mathbf{F}$

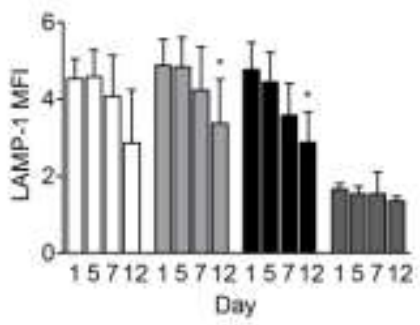

Plt fragments

\section{Supplementary figure S2.}

\section{Expression of PS and LAMP-1 on platelet subpopulations after agonist stimulation.}

The median fluorescence intensity (MFI) of PS (Annexin V-binding) and LAMP-1 in the different platelet subpopulations on day 1, 5, 7 and 12 of storage after stimulation with combinations of agonists; (A, D) CRP-XL+PAR1-AP, (B, E) CRP-XL+PAR4-AP, and (C, F) CRP-XL+PAR1-AP+PAR4-AP. Results are presented as mean \pm SD for $n=6$ except on day 5 and 12 where $\mathrm{n}=5$. $*=\mathrm{P}<0.05$ and $* *=\mathrm{P}<0.01$ compared to day 1 . PS-phosphatidylserine, LAMP-1-lysosome associated membrane protein-1, CRP-XL-cross-linked collagen related peptide, PAR1/4-AP-PAR1/4-activating peptide. 